\title{
Adiabatic invariance in volume-preserving systems
}

\author{
Anatoly Neishtadt ${ }^{1}$, Dmitri Vainchtein ${ }^{1,2}$ and Alexei Vasiliev ${ }^{1}$ \\ 1 Space Research Institute, Profsoyuznaya 84/32, Moscow 117810, Russia \\ aneishta@iki.rssi.ru, valex@iki.rssi.ru \\ 2 Georgia Institute of Technology, Atlanta, GA, USA \\ dmitri@gatech.edu
}

Summary. We consider destruction of adiabatic invariance in volume-preserving systems due to separatrix crossings, scattering on and capture into resonances. These mechanisms result in mixing and transport in large domains of phase space. We consider several examples of systems where these phenomena occur.

Key words: adiabatic invariant, separatrix, resonance

\section{Introduction}

Consider a volume-preserving system of ODE in $\mathbf{R}^{3}$ depending on a small parameter, $\varepsilon$ : $0<\varepsilon \ll 1$ :

$$
\dot{\mathbf{x}}=\mathbf{v}(\mathbf{x})+\varepsilon \mathbf{w}(\mathbf{x}, \varepsilon), \quad \operatorname{div} \mathbf{v}=\operatorname{div} \mathbf{w}=0 .
$$

Velocity field $\mathbf{v}$ in (1) defines an unperturbed (base) flow; $\mathbf{w}$ is a perturbation and is supposed to be a smooth function of $\varepsilon$. We restrict our discussion to 3-D autonomous flows, providing remarks on possible generalizations where applicable. System (1) at $\varepsilon=0$ is called the unperturbed system. Systems of kind (1) arise naturally in problems of incompressible fluid dynamics. Therefore, it is customary to call phase trajectories of (1) streamlines. A natural way to illustrate dynamics driven by (1) is to consider the motion of passive tracers advected by the flow. In a sense, passive tracers for the flows are equivalent to phase points in generic dynamical systems.

Let the unperturbed system be integrable. Then the phase space of the system (that for hydrodynamical flows coincide with the physical space) is foliated into invariant tori and the motion on these tori is quasi-periodic or periodic. If there are two independent integrals, the tori are invariant closed curves. In general, the integrability requires the existence of at least one conserved quantity (or action or invariant), so all flows of interest belong to one of two classes: action-action-angle or action-angle-angle [10]. Transport in the 
perturbed action-angle-angle flows is severely restricted by KAM tori (it was illustrated in [8]), while the effective degeneracy of the action-action-angle flows opens the possibility of global transport and mixing. We will, therefore, focus our attention on action-action-angle flows and possible mechanisms leading to chaotic advection.

The effects of the small perturbation in (1) start manifesting themselves on time intervals of order at least $\varepsilon^{-1}$. A function of phase variables is called an adiabatic invariant (AI) if its value along a phase trajectory of (1) has only small (with $\varepsilon$ ) variations on time intervals of such length. In other words, an AI is an approximate first integral of the system. Perpetual conservation of AI presents a barrier for complete mixing.

In this paper, we describe destruction of adiabatic invariants at separatrices and resonances and use several examples studied earlier [26, 18, 17, 23] to illustrate different aspects of the complete picture. General properties are discussed in Section 2. Separatrix crossings are discussed in Section 3, and passages through resonances are considered in Section 4.

\section{Separatrix crossings and passages through resonances in volume-preserving systems}

In this section, we give an introductory description of slow-fast volumepreserving systems with separatrix crossings and passages through resonances.

Let unperturbed system (1) be integrable and of the action-action-angle type. Then, almost the entire phase space is filled with closed phase trajectories. In terms of the two independent integrals of motion, $I$ and $J$, the unperturbed system can be expressed in the following form (so-called Nambu system [11]):

$$
\dot{\mathbf{x}}=\mu(I, J)[\operatorname{grad} I, \operatorname{grad} J],
$$

where the square brackets denote the vector product, and $\mu(I, J)$ is a function of values of the integrals $I$ and $J$ (in the examples of Sections 3.1, 3.2, $\mu \equiv 1$ ). A joint level of the two integrals $I=i, J=j$ defines a closed unperturbed phase trajectory $\Gamma_{i, j}$. Introduce on $\Gamma_{i, j}$ an angular variable $\phi \bmod 2 \pi$ changing at a constant rate in the unperturbed motion.

The perturbation in (1) causes the values of $i$ and $j$ to change at a rate of order $\varepsilon$ in the motion along a perturbed trajectory. In terms of the variables $i, j, \phi$, perturbed system (1) can be written as

$$
\frac{\mathrm{d} i}{\mathrm{~d} t}=\varepsilon f(i, j, \phi, \varepsilon), \quad \frac{\mathrm{d} j}{\mathrm{~d} t}=\varepsilon g(i, j, \phi, \varepsilon), \quad \frac{\mathrm{d} \phi}{\mathrm{d} t}=\omega(i, j)+\varepsilon h(i, j, \phi, \varepsilon) .
$$

The functions $f, g, h$ are $2 \pi$-periodic in $\phi$. In (3), the variables $i, j$ are "slow", and the variable $\phi$ is "fast". Define the averaged system:

$$
\frac{\mathrm{d} i}{\mathrm{~d} t}=\varepsilon F(i, j), \quad \frac{\mathrm{d} j}{\mathrm{~d} t}=\varepsilon G(i, j),
$$


where functions $F$ and $G$ are obtained by the averaging of $f$ and $g$, respectively, over $\phi$ :

$$
F(i, j)=\frac{1}{T(i, j)} \oint_{\Gamma_{i, j}}(\operatorname{grad} I, \mathbf{w}) \mathrm{d} t, \quad G(i, j)=\frac{1}{T(i, j)} \oint_{\Gamma_{i, j}}(\operatorname{grad} J, \mathbf{w}) \mathrm{d} t .
$$

In (5), $\mathbf{w}$ is calculated at $\varepsilon=0, T(i, j)$ is the period of the unperturbed motion along $\Gamma_{i, j}$, parentheses denote the scalar product, and the integration is performed along $\Gamma_{i, j}$. Far from singular surfaces (described below), solutions of the averaged system describe variations of $i, j$ in complete system (1) with the accuracy of order $\varepsilon$ on time intervals of order $\varepsilon^{-1}[5,2]$.

Let $\Phi(i, j)$ be the flux of the perturbation through a surface spanning $\Gamma_{i, j}$. Due to the preservation of the volume, the value of $\Phi(i, j)$ does not depend on a particular choice of the surface. A remarkable fact is that the averaged system is Hamiltonian, and $\Phi(i, j)$ is the Hamiltonian function (see, e.g., $[26,19])$ :

$$
\frac{\mathrm{d} i}{\mathrm{~d} t}=\frac{\varepsilon}{\mu(i, j) T(i, j)} \frac{\partial \Phi(i, j)}{\partial j}, \quad \frac{\mathrm{d} j}{\mathrm{~d} t}=-\frac{\varepsilon}{\mu(i, j) T(i, j)} \frac{\partial \Phi(i, j)}{\partial i} .
$$

It follows from $(6)$ that $\Phi(i, j)$ is an integral of the averaged system. Standard assertions about the accuracy of the averaging method (see, e.g., $[5,2,3]$ ) imply that $\Phi$ is an approximate integral of the motion in exact system (1), i.e., $\Phi$ is an adiabatic invariant. However, the averaging method breaks down in a neighborhood of 2-D singular surfaces. These surfaces can be of one of three types: (i) separatrix surfaces containing non-degenerate hyperbolic fixed points of the unperturbed system and filled by heteroclinic trajectories connecting them, (ii) separatrix surfaces containing a line of degenerate singular points (this case occurs, in particular, in 1 d.o.f. Hamiltonian systems depending on a slowly varying parameter, and it is considered elsewhere $[22,7,12])$, (iii) resonance surfaces, corresponding to closed curves filling a surface. In systems with one angle variable, the function $\omega(i, j)$ in (3) is identically zero everywhere on such surface. The major difference between separatrix and resonance surfaces is that near separatrix surfaces the base flow slows down only in the immediate vicinity of fixed points of the base system. In comparison, the base flow slows down everywhere near a resonance surface.

If system (1) possesses singular surfaces, dynamics can be described as follows. Far from singular surfaces, a phase point $i(t), j(t)$ closely (with the accuracy $\mathrm{O}(\varepsilon)$ ) follows a trajectory of the averaged system. The quantity $\Phi(i, j)$ along the streamline (phase trajectory) oscillates with an amplitude of order $\varepsilon$ around a certain constant value, say, $\Phi_{1}$. When the streamline crosses a small neighborhood of a singular surface, $\Phi$ changes by a value $\Delta \Phi$, that is in general much greater than $\varepsilon$. After this neighborhood is crossed, the value of $\Phi$ along the trajectory oscillates near a new constant value, $\Phi_{2}=\Phi_{1}+\Delta \Phi$. As the main change occurs in a narrow neighborhood of a singular surface, we shall call this change a jump of the AI. The magnitude of a jump turns out to be very sensitive to variations of initial conditions. Therefore, the jump is 
in a sense random. If allowed by the geometry of the system, the streamline comes to the separatrix again and the process repeats itself. Accumulation of jumps at multiple crossings results in destruction of the adiabatic invariance (i.e. the AI changes by a value of order 1) and leads to chaotic dynamics in the system.

A complete description of chaotic advection in these problems starts with a description of a single crossing of a resonance or a separatrix surface. We obtain an asymptotic formula for this change of the AI. In the case of crossing a resonance, we also study the possibility of capture into the resonance and describe the captured dynamics. Second, based on the equations for a single passage, we describe statistical properties of jumps and use them to study the long-time dynamics on time intervals that include many crossings. We show that there are two quantities that describe the chaotic advection over the long intervals of time that include many crossings: the size of the chaotic domain and a characteristic rate of mixing inside the chaotic domain. The regular domain is filled with streamlines that do not cross singular surfaces, while the domain of chaotic advection is filled with streamlines that repeatedly cross at least one singular surface. A technique to estimate the excess width of the chaotic domain was developed in [25]. The rate of mixing is given by the properties of the diffusion of the AI.

The accumulation of the jumps of AI leads to the diffusion across level surfaces of the AI. Mixing on a level of AI is much faster. Hence, the overall rate of mixing is defined by the diffusion of AI. Similar phenomena were observed in 3-D volume-preserving maps ([8]) and in Hamiltonian systems (see [24] and references therein).

\section{Separatrix crossings in volume-preserving systems}

In this section, we consider two examples of volume-preserving systems where separatrix crossings result in the destruction of adiabatic invariance to illustrate different aspects of the evolution. The problem in a more general setting was studied in [19]. Let us just briefly note that the problem of jumps of adiabatic invariants at separatrix crossings in volume-preserving systems cannot be reduced to similar problems in Hamiltonian systems depending on a slowly varying parameter $[22,7,12]$, or in slow-fast Hamiltonian systems [13]. Although ideologically close to them, this problem needs an independent study.

\subsection{Example 1: quadratic Stokes flow in the unit sphere}

Consider the volume-preserving system

$$
\begin{aligned}
& \dot{x}=-8 x y+\varepsilon z, \\
& \dot{y}=11 x^{2}+3 y^{2}+z^{2}-3, \\
& \dot{z}=2 z y-\varepsilon x .
\end{aligned}
$$


This system was introduced in [4] as an example of a hydrodynamic flow with chaotic advection [1] and in connection with "fast dynamo problem" in magneto-hydrodynamics [27, 29]. System (7) satisfies the Stokes equation for a flow of a viscous fluid. The flow can be regarded as a composition of three flows, namely Hill's spherical vortex, a quasi-rigid rotation, and a twist flow.

The unit sphere is invariant under the flow of system (7), and here we discuss only dynamics inside the sphere. For $\varepsilon=0$, the system is integrable. The numerical simulations seem to show that at arbitrarily small $\varepsilon \neq 0$ the whole interior of the unit sphere, at least up to a small measure, is a domain of chaotic motion.

Consider the unperturbed system (see Fig. 1a). The explicit form for the integrals $I$ and $J$ was obtained in [4]:

$$
I=x z^{4}, \quad J=\frac{x^{2}+y^{2}+z^{2}-1}{z^{3}} .
$$

The poles of the unit sphere $\mathrm{N}$ and $\mathrm{S}(x=z=0, y= \pm 1)$ are singular points of the flow. Heteroclinic trajectories going from $\mathrm{N}$ to $\mathrm{S}$ fill the part of the plane $x=0$ lying inside the unit sphere. We shall call this surface the separatrix $\mathcal{S}$ of the unperturbed system. Two heteroclinic trajectories $h_{1}: z=0, x^{2}+y^{2}=1, x<0$ and $h_{2}: z=0, x^{2}+y^{2}=1, x>0$ go from $\mathrm{S}$ to $\mathrm{N}$. All the other trajectories are closed curves.

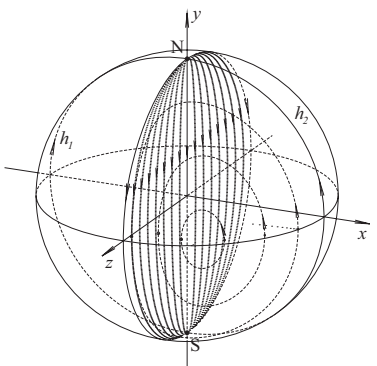

(a)

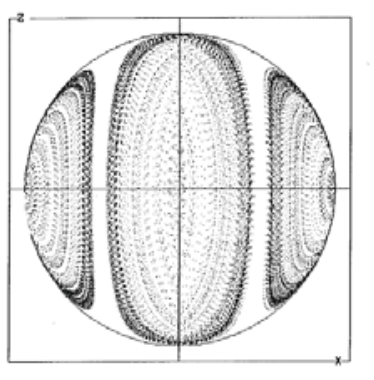

(b)

Fig. 1. (a): The unperturbed flow. (b): Result of long time integration of a phase trajectory giving evidence of the chaotic behavior. $\varepsilon=0.1$.

For $\varepsilon \neq 0$, system (7) is no longer integrable. Integrals (8) are not preserved. Streamlines are not closed and cross the separatrix $\mathcal{S}$. Figure 2a represents a result of long integration of one perturbed phase trajectory. The dots mark the points where the trajectory crosses the equatorial plane. The points tend to fill the interior of the unit circle in $(x, z)$-plane, which is an evidence of chaotic behavior in the system.

Dynamics of the perturbed system is presented in Fig. 2 as a segment of a typical streamline and the phase portrait of the averaged system on the $(j, i)$ 


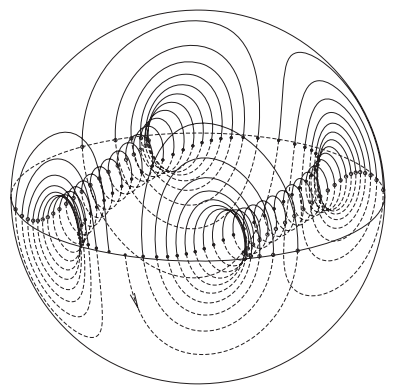

(a)

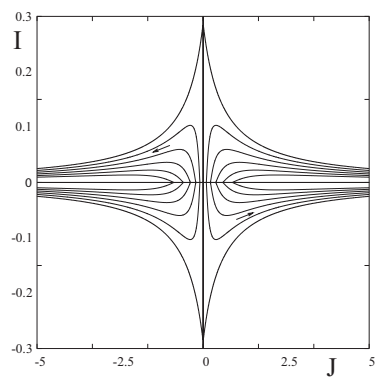

(b)

Fig. 2. Dynamics of the perturbed system over one long period. (a): A perturbed streamline. (b): Phase portrait of the averaged system.

plane. In Fig. 2b, the curves are level lines of $\Phi$. The separatrix corresponds to the line $i=0$. The departure of the trajectories to infinity in $j$ is a fictitious singularity associated with the particular choice of variables $I$ and $J$. The motion along trajectories of the averaged system is periodic with period of order $\varepsilon^{-1}$. On each period, each trajectory crosses the separatrix twice.

Along a phase trajectory of (7) far from $\mathcal{S}$, the value of $\Phi$ undergoes oscillations of order $\varepsilon$. Near $\mathcal{S}$ (at $|i| \ll 1$ ), the accuracy of the averaging method breaks down, and a more accurate consideration is necessary. The behavior of $\Phi$ along a phase trajectory in this region is described in [26] and [17], and here we present only the main results.

Consider a segment of a phase trajectory $\gamma$ of the perturbed system that crosses $\mathcal{S}$ once. Let $M_{-}$and $M_{+}$be its initial and final points lying at a distance of order 1 from $\mathcal{S}$. Denote by $\Phi_{ \pm}$the values of $\Phi(i, j)$ at the points $M_{ \pm}$. Then for $\Delta \Phi=\Phi_{+}-\Phi_{-}$in the main approximation the following formula holds:

$$
\Delta \Phi=-\varepsilon^{3 / 4}\left|\Theta\left(j_{0}\right)\right|^{3 / 4} a\left|j_{0}\right| \frac{1}{\Gamma(1 / 4)} \int_{0}^{\infty} \frac{t^{-3 / 4}\left(\mathrm{e}^{-\xi t}-\mathrm{e}^{-(1-\xi) t}\right)}{1-\mathrm{e}^{-t}} \mathrm{~d} t .
$$

Here

$$
\Theta(j)=\oint_{\Gamma_{0, j}}(\operatorname{grad} I, \mathbf{w}) \mathrm{d} t, \quad a=-\frac{3 \sqrt{\pi}}{8} \frac{\Gamma(5 / 8)}{\Gamma(9 / 8)},
$$

where $\Gamma(\cdot)$ is the Gamma function, and the value $j_{0}$ is defined below. The value of $\xi$ is a function of the initial conditions defined as follows. Assume for definiteness that $\gamma$ crosses $\mathcal{S}$ at $z>0(j<0)$. The trajectory $\gamma$ of the perturbed system is a spiral. Consider the points on $\gamma$ where $\dot{y}=0, y>0$ (the uppermost points on the turns of the spiral). Denote the last of them before $\mathcal{S}$ crossing as $M_{0}$. Let $i_{0}, j_{0}$ represent values of $I, J$ at $M_{0}$. By definition $i_{0} \leq 0$. It is possible to show that $i_{0}>-\varepsilon \Theta_{0}-C \varepsilon^{7 / 4}$, where $C=$ const $>0, \Theta_{0}=\Theta\left(j_{0}\right)$ (because during the turn $\gamma_{0}$ the value $i$ grows approximately by $\varepsilon \Theta_{0}$ ). Then 
we define $\xi=-i_{0} /\left(\varepsilon \Theta_{0}\right)$. Formula (9) holds if $c_{1} \varepsilon^{3 / 4}<\xi<1-c_{1} \varepsilon^{3 / 4}$, where $c_{1}$ is a positive constant.

The quantity $\xi$ strongly depends on the initial conditions. A small, of order $\varepsilon$, variation in values of $i, j$ at $M_{-}$produces, in general, variation of order 1 in the value of $\xi$. It can be shown that $\xi$ can be treated as a random value uniformly distributed on $(0,1)$. Accordingly, $\Delta \Phi$ is also treated as a random value with statistical properties defined by (9). The jumps in the AI result in chaotization of the dynamics inside the unit sphere. It is natural to suppose that the system is ergodic inside the sphere (at least up to a residual of a small measure). This hypothesis can be checked numerically. Figure 3a represents the plot of distribution of jumps of the AI found numerically after $10^{8}$ separatrix crossings, and the theoretical distribution function obtained using (9) under the hypothesis of ergodicity. One can see a remarkably good agreement.
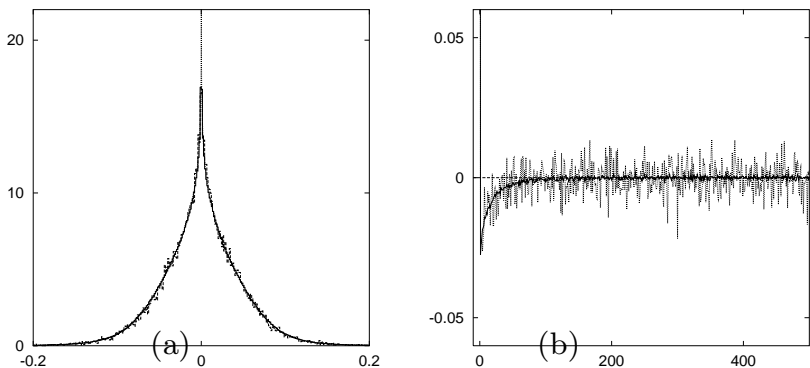

Fig. 3. (a) Distribution of jumps of the adiabatic invariant, $\varepsilon=0.1$ (a histogram). The dotted line represents the theoretical distribution. (b) Autocorrelation function of the jumps in $\Phi$ at $\varepsilon=0.1$. The broken and the solid lines are calculated using $10^{6}$ and $10^{8}$ jumps, respectively. The plots are obtained by C. Simó [17].

The motion in the system can be described as follows (cf. Fig. 2). Far from $\mathcal{S}$, a phase point $i(t), j(t)$ closely (with the accuracy $\mathrm{O}(\varepsilon)$ ) follows a trajectory of the averaged system. In other words, the value of $\Phi(i, j)$ along the phase trajectory oscillates with an amplitude of order $\varepsilon$ around a certain constant value, say, $\Phi_{1}$. When the trajectory crosses a small neighborhood of $\mathcal{S}$, value of $\Phi$ undergoes a quasi-random jump of order $\varepsilon^{3 / 4}$. After this neighborhood is crossed, value of $\Phi$ along the trajectory is again close to a constant value $\Phi_{2}$, and the difference $\Phi_{2}-\Phi_{1}$ is given by (9). After the next separatrix crossing, value of the $\mathrm{AI}$ changes again, and so on.

Consider statistical properties of the jumps in $\Phi$ along one phase trajectory of the system. Let two successive separatrix crossings be characterized by values $\xi_{1}$ and $\xi_{2}$. A small variation $\delta \xi_{1}$ in $\xi_{1}$ produces a variation of the jump in $\Phi$ by $\sim \varepsilon^{3 / 4} \delta \xi_{1}$. In the period of time $\sim \varepsilon^{-1}$ before the next crossing, it results in variation $\sim \varepsilon^{-1 / 4} \delta \xi_{1}$ of the phase $\phi$ of the point. Hence, the value $\xi_{2}$ obtains a variation $\delta \xi_{2} \sim \varepsilon^{-1 / 4} \delta \xi_{1}$, and $\delta \xi_{2} / \delta \xi_{1} \sim \varepsilon^{-1 / 4} \gg 1$. Therefore, it is natural 
to suppose that $\xi_{1}$ and $\xi_{2}$ are statistically independent and the successive jumps in $\Phi$ are not correlated. In this case, variation of the AI along a phase trajectory can be modeled as a random walk. It takes a time of order $\varepsilon^{-5 / 2}$ for the AI to change by a value of order 1 . To test the assumption about the independence of successive jumps, the autocorrelation function of the jumps in $\Phi$ was computed. The numerical results shown in Fig. 3b demonstrate fast decay of the autocorrelation function.

\subsection{Example 2: Chaotic advection in a cubic Stokes flow}

The flow in this example was introduced in [21] as a flow inside a neutrally buoyant spherical drop immersed in a linear flow. In spherical coordinates, the velocity field of the flow takes the form:

$$
\begin{aligned}
\frac{d r}{d t} & =\frac{3}{4} r\left(r^{2}-1\right)\left(1-3 \cos ^{2} \theta\right), \\
\frac{d \theta}{d t} & =\frac{3}{4}\left(5 r^{2}-3\right) \sin \theta \cos \theta-\frac{1}{2} \varepsilon \omega_{x} \sin \varphi, \\
\frac{d \varphi}{d t} & =\frac{1}{2} \varepsilon\left(\omega_{z}-\omega_{x} \cot \theta \cos \varphi\right),
\end{aligned}
$$

where $0<\varepsilon \ll 1$ is a small parameter, and $\omega_{x} \geq 0, \omega_{z} \geq 0$ are the components of vorticity vector $\omega,|\omega|=1$. Flow (10) is a superposition of two flows: a slow rotation at the angular velocity $\frac{1}{2} \varepsilon \omega$ and a flow that deforms fluid elements, defined by the terms in (10) that are independent of $\omega$. It was demonstrated in [21] that flow (10) may possess chaotic streamlines.

The unperturbed flow, i.e. system (10) at $\varepsilon=0$, (see Fig. 4 a) possesses two independent integrals of motion and, thus, is integrable. Almost all streamlines of the unperturbed flow are closed:

$$
\psi=\frac{3}{4} r^{3}\left(r^{2}-1\right) \sin ^{2} \theta \cos \theta .
$$

The level surfaces of integral $\varphi$ are the half-planes containing the $z$-axis as their border. The level surfaces of integral $\psi$ form two families of nested tori filling the northern and the southern hemispheres of the unit sphere. Almost all the streamlines of the unperturbed flow are closed curves. Besides the closed streamlines, there are also heteroclinic streamlines connecting fixed points of the flow. The unperturbed flow has the following fixed points: two saddle points in the poles of the sphere, a saddle point at the origin, a family of degenerate fixed saddle points filling the equator, and two families of degenerate elliptic fixed points filling the circles $\psi= \pm 3 /\left(5^{5 / 2}\right)$. There are two heteroclinic trajectories connecting the origin to the poles of the sphere, two families of heteroclinic trajectories filling the surface of the sphere and connecting its poles to the equator, and a family of heteroclinic trajectories filling the equatorial plane $z=0, x^{2}+y^{2}<1$ and connecting the degenerate 
saddle points on the equator to the origin. We shall call the part of equatorial plane $z=0, x^{2}+y^{2}<1$ (or $\psi=0$ ) the separatrix, $\mathcal{S}$.

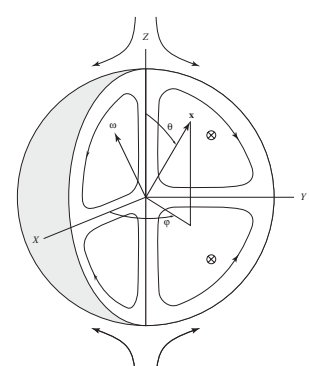

(a)

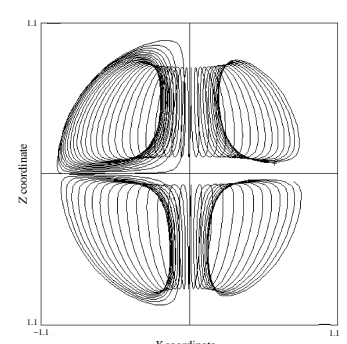

(b)

Fig. 4. (a) The unperturbed flow, $\varepsilon=0$. (b) A segment of a phase trajectory in the perturbed system, $\varepsilon \neq 0$.

Assume that $\omega_{x} \neq 0$. A segment of a trajectory of the perturbed system that crosses $\mathcal{S}$ once is shown in Fig. 4b. As above, we introduce the function $\Phi$ and the averaged system. On $\mathcal{S}$, the averaged system is not defined and $\Phi$ is discontinuous. To make it continuous, we introduce $\tilde{\Phi}=\Phi+\omega_{z} \operatorname{sign}(\psi) / 6$. From now on, the tilde is omitted. Phase portraits of the averaged system are presented in Fig. 5. If $\omega_{z} / \omega_{x}>1 / \sqrt{2}$, there are regions on the phase portrait (see Fig. 5a) filled with phase trajectories that do not cross $\mathcal{S}$. These regions contain the streamlines of (10), surrounding the circles $\psi= \pm 3 / 5^{5 / 2}$. If $\omega_{z} / \omega_{x}<1 / \sqrt{2}$ (see Fig. 5 b), all phase trajectories of the averaged system $\operatorname{cross} \mathcal{S}$.

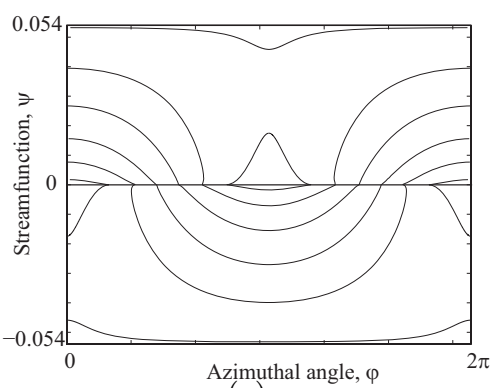

(a)

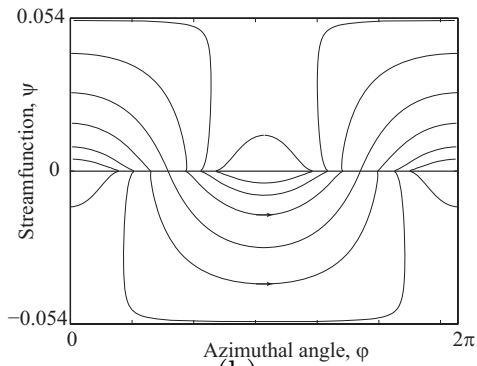

(b)

Fig. 5. Phase portraits of the averaged system. (a) $\omega_{z} / \omega_{x}=0.8$. (b) $\omega_{z} / \omega_{x}=0.5$.

The jump in the AI at the separatrix crossing is given in the main approximation by (see [18]): 


$$
\Delta \Phi=\sqrt{\varepsilon} a \cos \varphi_{0} \omega_{x}\left|\Theta_{0}\right|^{1 / 2} \frac{1}{\sqrt{\pi}} \int_{0}^{\infty} \frac{t^{-1 / 2}\left(e^{-\xi t}-e^{-(1-\xi) t}\right)}{1-e^{-t}} \mathrm{~d} t .
$$

In (12), $a=(6 \pi)^{-1 / 2}(\Gamma(3 / 4))^{2}, \Theta_{0}=-\frac{1}{6} \omega_{x} \sin \varphi_{0}, \xi=-\psi_{0} /\left(\varepsilon \Theta_{0}\right), \xi \in$ $(0,1) ; \varphi_{0}$ and $\psi_{0}$ are values of $\varphi$ and $\psi$ at the point $M_{0}$ defined as follows. Consider a segment of a perturbed streamline that crosses $\mathcal{S}$ once. It is shaped as a spiral (see Fig. 4b). On each turn of the spiral, mark the point where $\dot{r}=0, r<1 / 2$ (the closest to the origin point of the turn). Thus, $M_{0}$ is the last of these points prior to the separatrix crossing.

As in the previous example, the value of $\xi$ is highly sensitive to the initial conditions and should be treated as a random variable uniformly distributed on the interval $(0,1)$. Values of $\xi$ for two successive separatrix crossings are statistically independent, at least for a majority of phase trajectories.

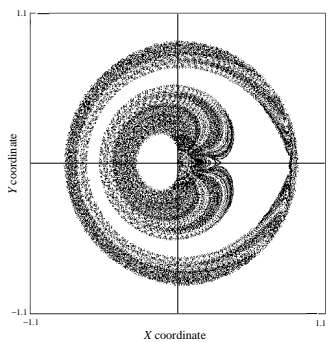

(a)

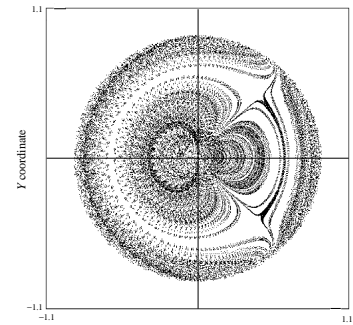

(c)

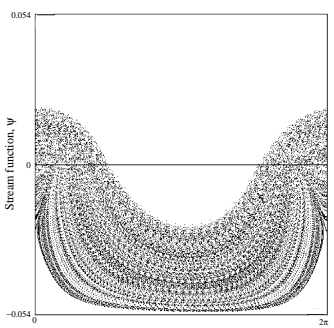

(b)

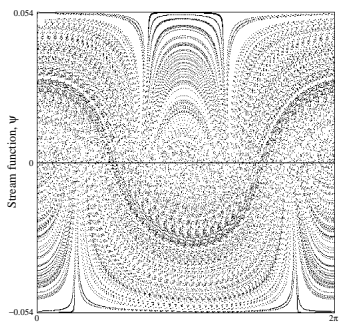

(d)

Fig. 6. Representation of a long phase trajectory of system (10), $\varepsilon=0.05$. (a),(b) $\omega_{z} / \omega_{x}=0.8$. (c),(d) $\omega_{z} / \omega_{x}=0.5$. (a),(c) Sections by the plane $z=1 / \sqrt{5}$, (b),(d) Projections on the $(\phi, \psi)$-plane.

The accumulation of the jumps of AI leads to diffusion of the AI. In time $t \sim \varepsilon^{-2}$, the value of the AI along a trajectory varies by a quantity of order 1 . Long-time dynamics in the system is illustrated in Fig. 6. In order to represent a long phase trajectory, we used its Poincaré sections by the planes $z= \pm 1 / \sqrt{5}$ and a projection on the $(\psi, \varphi)$ plane. Each dot on the plane corresponds to 
one crossing of one of the planes $z= \pm 1 / \sqrt{5}$ taking place at $r>\sqrt{3 / 5}$. The time of calculation corresponds approximately to 1000 separatrix crossings, and the diffusion of the AI, both as the intermittent character of motion can be clearly seen. One can see in Figs. 6a, b, that the domain of chaotic motion coexists with the domain of regular motion not visited by the trajectory (in Fig. $6 \mathrm{~b}$ it is the domain below the area filled with dots; the domain above is not visited by the trajectory due to the significant decrease in the diffusion rate at the line $\Phi(\psi, \varphi)=0$, see details in [18]). In Figs. 6c, d, one can see that all the interior of the unit sphere is the domain of chaotic dynamics.

\section{Passages through resonances in volume-preserving systems}

In the current section we discuss another type of phenomena that occur at singular surfaces: scattering on and capture into resonance. In what follows, we will consider 3-D autonomous flows of the action-action-angle type. However, let us note that the resonance phenomena take place also in systems with two fast phases (angles) and two actions (e.g., systems with time-dependent external forcing, similar to those appearing in $[6,20,28]$ ). Such systems can be considered along the same lines, because partial averaging near the resonance transforms these systems to the action-action-angle form (see [16]).

\subsection{Example: a Taylor-Couette flow between two cylinders}

As an example, we consider a volume-preserving kinematic model inspired by a Stokes Taylor-Couette flow between two infinite counter-rotating coaxial

cylinders (the "vertical" $z$ axis is along the axis of the cylinders, $\rho$ is the distance from the axis and $\theta$ is an angle in the "horizontal" plane) (see [23] for a complete description). In the dimensionless units, the flow is

$$
\begin{aligned}
\dot{\rho} & =\varepsilon \kappa(\rho-1) \cos \theta, \\
\dot{z} & =\varepsilon(1+\ln \rho / \ln \eta), \\
\dot{\theta} & =\omega(\rho, z)-\frac{1}{\rho} \varepsilon \kappa(2 \rho-1) \sin \theta .
\end{aligned}
$$

The value of $\rho$ changes between $\rho=1$ (at the inner cylinder) and $\rho=1 / \eta$ (at the outer cylinder). The frequency of the unperturbed flow, $\omega(\rho, z)$, is

$$
\omega(\rho, z)=-\rho \frac{\eta}{1-\eta}+\frac{1}{\rho} \frac{1}{1-\eta}+\frac{\eta}{1-\eta^{2}} \delta \sin (\lambda z)\left(\rho-\frac{1}{\rho}\right),
$$

where $\lambda=2 \pi$ and $\delta$ are the wavenumber and amplitude of oscillations of the frequency of the outer cylinder, respectively. One can see that $\omega=1$ and $\omega=-1+\delta \sin (\lambda z)$ on the inner and the outer cylinders, respectively. 
One can imagine the outer cylinder to consist of rings, each of which rotates with its own speed (see Fig. 7a). The variables $\rho$ and $z$ are the integrals of the unperturbed system. The unperturbed streamlines are circles with the direction of the rotation depending on the sign of $\omega(\rho, z)$.

The perturbation consists of two parts. The first is a vertical (in the axial direction) shear-type flow (the $\dot{z}$ term). The second is an additional angular rotation due to a slight non-circularity of the outer cylinder. In (13), $0 \leq \varepsilon \ll$ 1 is a small parameter, while $\kappa \sim 1$ defines a characteristic ratio of the two perturbations. The axial velocity, $\dot{z}$, equals $\varepsilon$ at $\rho=1$ and vanishes at $\rho=1 / \eta$.

For $\varepsilon>0$, the variable $\theta$ is fast and the variables $\rho$ and $z$ are slow. Thus, we can average (13) over one period of $\theta$. The averaged trajectories (in the full 3 -D, $(\rho, z, \theta)$, space) spiral around the cylinders of constant radius ( $\rho=$ const) with the direction of the rotation depending on the sign of $\omega$. The averaged system is Hamiltonian and a quantity $\Phi$,

$$
\Phi=\rho,
$$

is a Hamiltonian. It is an integral of the averaged system and is an adiabatic invariant of the exact system. The averaging is valid away from a resonance surface (in 3-D), or a curve on the slow, $(\rho, z)$-plane where $\omega=0$. We denote that surface by $\mathrm{R}$. It follows from (14) that R is given by

$$
\rho_{R}^{2}(z)=\frac{1}{\eta} \frac{1+\eta-\eta \delta \sin (\lambda z)}{1+\eta-\delta \sin (\lambda z)}
$$

and located between $\rho_{\min }$ and $\rho_{\max }$. The division of the flow domain is shown in Fig. $7 \mathrm{~b}$ (note that we plotted $z \bmod 1$ ). Trajectories to the left and to the right of the corresponding vertical lines do not cross $\mathrm{R}$.

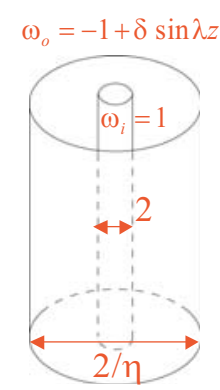

(a)

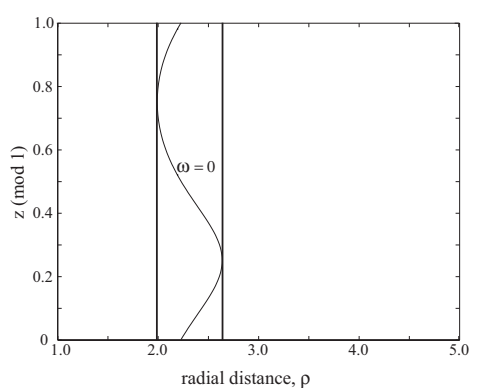

(b)

Fig. 7. (a) Flow structure for the unperturbed system. (b) Division of the flow domain for $z$ mod 1. A chaotic domain is between the vertical lines. A regular (KAM) domain consists of two parts at the left and at the right. The wavy line in the middle is the resonance, $\delta=0.4, \eta=0.2$. 
As a passive tracer approaches $\mathrm{R}$, it can be either scattered at a resonance or captured into resonance. While the scattering on resonance is somewhat similar to what happens at the separatrix crossing, capture can occur only at a resonance. Qualitatively, the difference between the two regimes can be described as follows. In the case of capture, upon arrival into the resonant zone, the phase switches its behavior from rotation to oscillation. The system drifts along the resonant surface for a long, of order $\varepsilon^{-1}$, time. As a result, the value of the AI changes by $\Delta \Phi=\mathrm{O}(1)$. Among all the streamlines that arrive to the resonant zone during a given time interval (of order $\varepsilon^{-1}$ ), only a small $\left(\mathrm{O}\left(\varepsilon^{1 / 2}\right)\right)$ part of streamlines are captured. In the case of scattering there is no phase oscillation. The streamlines pass through the resonance zone in an $\mathrm{O}\left(\varepsilon^{-1 / 2}\right)$ time and the corresponding jump in the $\mathrm{AI}$ is $\Delta \Phi=\mathrm{O}\left(\varepsilon^{1 / 2}\right)$. We describe these two processes below.

\section{Scattering on resonance}

In the vicinity of $\mathrm{R}$, dynamics is defined in terms of $\theta$ and a coordinate, $\sigma=\sigma\left(\rho_{R}(z), z\right)$, along $\mathrm{R}$ chosen in such a way that the infinitesimal volume is $d V=d \sigma d \omega d \theta$ :

$$
\dot{\sigma}=\varepsilon f_{2,0}, \quad \ddot{\theta}=\varepsilon\left(a+b_{1} \cos (\theta)\right)-\varepsilon \frac{\partial f_{2,0}}{\partial \sigma} \dot{\theta},
$$

where

$$
\begin{aligned}
a & =\frac{\eta}{1-\eta^{2}} \delta \lambda \cos (\lambda z)\left(\rho-\frac{1}{\rho}\right)(1+\ln \rho / \ln \eta), \quad b_{1}=-2 \kappa \frac{1}{\rho+1}, \\
f_{2,0} & =-\frac{1}{2} \rho\left(\rho^{2}-1\right)(1+\ln \rho / \ln \eta) .
\end{aligned}
$$

In the case of scattering, we can consider the value of $\sigma$ (or $z$ and $\rho$ ) in (16) fixed and the dynamics is described by the resonance potential $V=$ $-a \theta-b_{1} \sin \theta$. The shape of phase portraits for the motion in the potential $V$ depends on the relation between $a$ and $b_{1}$. If

$$
\left|b_{1}\right|>|a|,
$$

the phase portrait looks like the one shown in Fig. 8a, and vice-versa for Fig. 8b.

In the process of scattering, the value of $\Phi$ undergoes a jump, the magnitude of which is (in the main approximation) given by

$$
\Delta \Phi=-2 s \sqrt{\varepsilon} \kappa \frac{\rho-1}{\sqrt{|a|}} \int_{s \infty}^{\bar{\theta}_{*}} \frac{\cos \theta}{\sqrt{2\left|s 2 \pi \xi+\theta+\left(b_{1} / a\right) \sin \theta\right|}} d \theta
$$

where $\bar{\theta}_{*}$ is the value of $\theta$ at the crossing, $s=\operatorname{sign}(a)$, and $\xi=$ $\left\{V\left(\theta_{*}\right) /(2 \pi|a|)\right\} \in(0,1)$, where the curly brackets denote the fractional part. If (17) holds, the ensemble average of $\Delta \Phi$ is: 


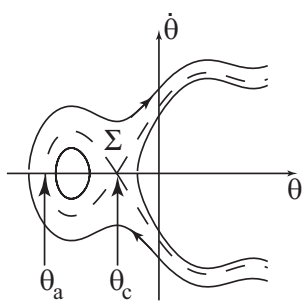

(a)

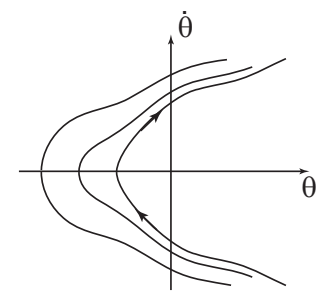

(b)

Fig. 8. Schematic phase portraits on the $(\theta, \dot{\theta})$ plane.
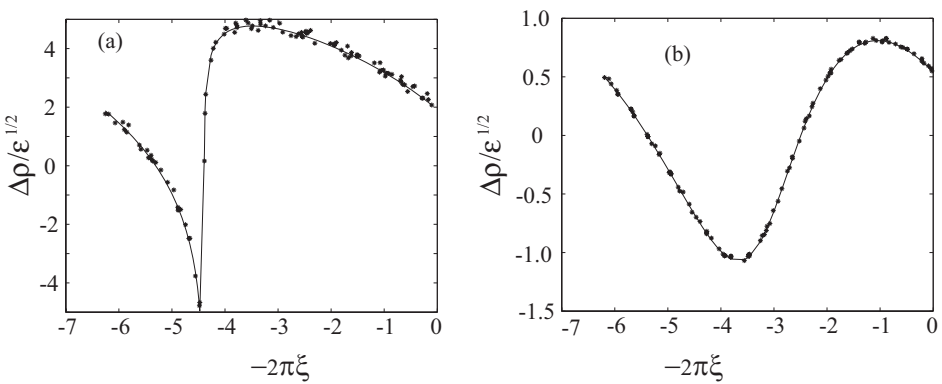

Fig. 9. The plot of $\Delta \rho / \sqrt{\varepsilon}$ as a function of $\xi$; (a): $\kappa=2$ and (b): $\kappa=0.2$. Note the difference in scales.

$$
\langle\Delta \Phi\rangle=-s \sqrt{\varepsilon} \frac{\rho^{2}-1}{2 \pi} S_{R} .
$$

Here $S_{R}$ is the area under the separatrix loop, $\Sigma$, in Fig. 8a:

$$
S_{R}=2\left|\int_{\theta_{a}}^{\theta_{c}} \sqrt{-2\left(V-V_{c}\right)} d \theta\right|,
$$

where $V_{c}$ is the value of $V$ at the hyperbolic fixed point in Fig. 8a. If (17) does not hold, $\langle\Delta \Phi\rangle=0$, as there is no separatrix, $S_{R}=0$.

Equation (18) was checked numerically for various values of parameters $\xi, \kappa$ and $\varepsilon$. In Fig. 9, the plots of $\Delta \rho(\xi) / \sqrt{\varepsilon}$ are presented for (a) $\kappa=2$ (when (17) is satisfied) and (b) $\kappa=0.2$, (when (17) is not satisfied). The solid lines in Fig. 9 correspond to theoretical values of $\Delta \rho(\varepsilon) / \sqrt{\varepsilon}$ and the asterisks show values obtained numerically from (13) for various values of $\xi$. When (17) is satisfied, $\Delta \rho(\xi)$ has a singularity.

\section{Capture into resonance}

The other phenomenon that affects the behavior of streamlines at a resonance crossing is capture into resonance. 

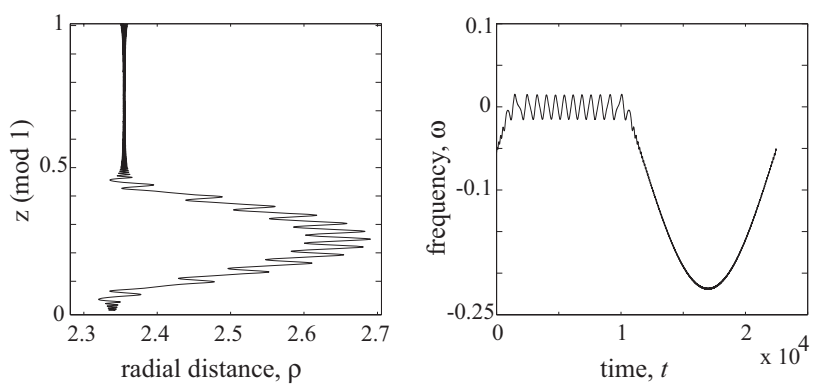

Fig. 10. Captured motion. (a) A projection of a streamline on the slow, $(\rho, z)$, plane; and (b) the time evolution of $\omega(\rho, z), \varepsilon=10^{-4}, \kappa=2$.

Capture into resonance is possible only if the phase portrait in the $(\theta, \dot{\theta})$ plane looks like the one shown in Fig. 8a, in other words, if there is a separatrix in the $(\theta, \dot{\theta})$-plane. Let $\Pi(0, \sigma)=f_{2,0} S_{R}$ be a flux of $f_{2,0}$ through the separatrix loop in Fig. 8a. Supposing that $\Pi(0, \sigma)$ increases as a phase point moves along a streamline, if a streamline comes very close to the hyperbolic fixed point, it may cross $\Sigma$ and, as a result, be caught in the oscillatory domain within the separatrix loop. In this case, a streamline starts shadowing the resonant surface. The captured motion is integrable and Hamiltonian. Depending on the structure of resonance, a tracer can be released from resonance (which is the case in the system under consideration) or reach the boundary of the system.

The dynamics of a typical capture is shown in Fig. 10 as a projection on the slow, $(\rho, z)$, plane and the time evolution of $\omega(\rho, z)$. A streamline comes from the bottom in Fig. 10a (from the left in Fig. 10b), is captured near $z=0.05(t=100)$, moves along the resonance, is released from the resonance near $z=0.45(t=1000)$, and then proceeds along an adiabatic path.

As it was discussed in $[14,15,9]$, capture can be considered as a probabilistic phenomenon: initial conditions for streamlines that are or are not captured are mixed. Consider a point $M$ far from the resonance such that streamline passing through $M$ intersects the resonance. Let $V^{d}$ be a sphere of radius $d$ centered at $M$ and $V_{c}^{d, \varepsilon}$ be the part of $V^{d}$ formed by initial conditions of trajectories with a capture into the resonance (see [14, 15, 9]). We define the probability of capture for the streamlines starting inside a small ball centered at $M$ as

$$
P(M)=\sqrt{\varepsilon} \lim _{d \rightarrow 0} \lim _{\varepsilon \rightarrow 0} \frac{\operatorname{vol} V_{c}^{d, \varepsilon} / \sqrt{\varepsilon}}{\operatorname{vol} V^{d}} .
$$

Following [15], we have:

$$
P(M)=\sqrt{\varepsilon} \frac{(\partial \Pi(0, \sigma) / \partial \sigma)_{*}}{2 \pi|a|_{*}} \sim \sqrt{\varepsilon}, \quad \text { if }\left(\frac{\partial \Pi(0, \sigma}{\partial \sigma}\right)_{*}>0,
$$




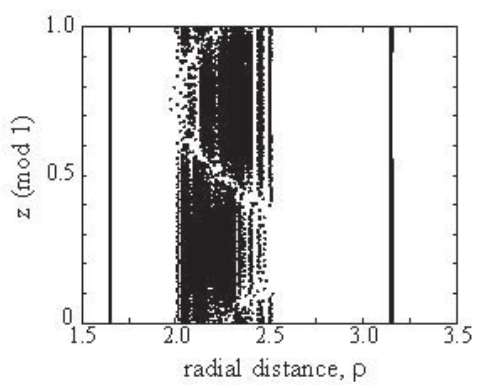

Fig. 11. Regular and chaotic domains with $z \bmod 1$. Almost straight vertical lines are regular streamlines. A single streamline that starts at $\rho_{\text {in }}=2.25$ fills almost the entire chaotic domain.

where the subscript ' $*$ ' indicates that the corresponding quantity must be evaluated when a given streamline comes to the resonance. For $(\partial \Pi(0, \sigma) / \partial \sigma)_{*}<$ $0, P(M)=0$.

\section{Long-time dynamics and adiabatic diffusion}

It was shown in Sect. 3 that the accumulation of the effects of the separatrix crossings leads to the destruction of the adiabatic invariance and chaotic advection. In the present section, we discuss similar phenomena in the volumepreserving flows with resonances.

It was shown above that the chaotic domain is a cylinder between $\rho_{\min }$ and $\rho_{\max }$ values, that depend on the parameters of the unperturbed system, $\eta$ and $\delta$, and are independent of the magnitude of perturbation, $\varepsilon$. Thus, the size of the chaotic domain is on the scale of the whole system regardless of how small $\varepsilon$ is. Outside the chaotic domain, the majority of streamlines is regular. A projection of three representative streamlines on the slow, $(\rho, z)$, plane is shown in Fig. 11. Almost straight vertical lines are regular streamlines. A single streamline that starts at $\rho_{i n}=2.25, z_{i n}=0, \theta_{i n}=0$ fills almost the entire chaotic domain.

We performed a set of numerical simulations to study the diffusion of adiabatic invariant and large scale mixing. 1000 initial conditions were uniformly distributed in a box $\rho_{i n} \times z_{i n} \times \theta_{i n}=[2.249,2.251] \times[-0.01,0.01] \times[-0.01,0.01]$ and we considered the Poincaré section located at $z=0.25 \bmod 1$. Every streamline crosses the resonance twice between the consecutive sections. A total change in $\rho$ after two successive crossings has zero mean.

Denote by $\Psi(\rho, N)$ the number of trajectories that, after $N$ double crossings, have the value of $\rho$ between $\rho-0.005$ and $\rho+0.005$. The spreading of $\Psi(\rho, N)$, obtained by integrating (13) with the initial conditions specified above, is shown in Fig. 12a. The second moment $\sigma^{2}(\Psi(\rho, N))=\left\langle\left(\rho-\rho_{0}\right)^{2}\right\rangle$ is presented in Fig. 12b. The constant slope confirms the diffusion assumption 


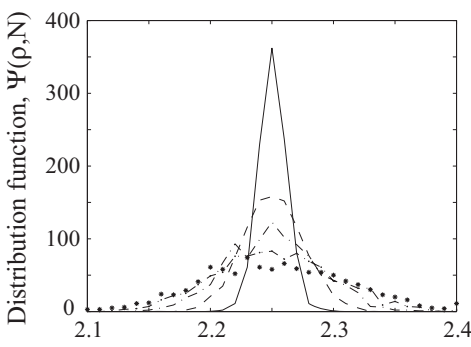

(a)

(b)

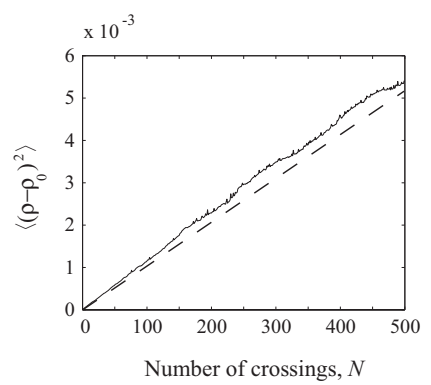

Fig. 12. Evolution of $\Psi(\rho, N)$ (a) The histogram of $\Psi$ for different $N$. The solid line and the stars correspond to $N=10$ and $N=400$, respectively. The other curves are between those values of $N$. (b) The square of the standard deviation, $\sigma^{2}(\Psi)$, averaged over 1000 trajectories for different $N$. The dashed line is a theoretical prediction $\sigma^{2}(\Psi)=2 D N$ with $D=D(\rho=2.25)=5.1 \times 10^{-6}$.

and the magnitude of the slope is in the good agreement with the prediction based on the simplified diffusion equation with $D(\rho)=D(\rho=2.25) \approx$ $5.1 \times 10^{-6}$, where $D(\rho)$, called the coefficient of the adiabatic diffusion, is given by the dispersion of $\Delta \rho$ :

$$
D(\varepsilon, \rho)=\varepsilon \widetilde{D}(\rho)=\int_{0}^{1}(\Delta \rho(\xi)-\langle\Delta \rho\rangle)^{2} d \xi
$$

\section{Acknowledgements}

The work was partially supported by RFBR Grants 06-01-00117 and NSh 13.12.2006.1. This material is based upon work partially supported by the National Science Foundation under Grant No. 0400370 and ITR-NSF Grant ACI-0086061. We are grateful to our co-authors Carles Simó and Igor Mezic.

\section{References}

1. H. Aref. Stirring by chaotic advection. J. of Fluid Mechanics, 143:1-21, 1984.

2. V.I. Arnold. Geometrical Methods in the Theory of Ordinary Differential Equations. Springer-Verlag, New York-Heidelberg-Berlin, 1983.

3. V.I. Arnold, V.V. Kozlov, and A.I. Neishtadt. Dynamical Systems III. Encyclopedia of Mathematical Sciences. Springer-Verlag, New York, N.Y., 1988.

4. K. Bajer and H.K. Moffatt. On a class of steady confined Stokes flows with chaotic streamlines. J. of Fluid Mechanics, 212:337-363, 1990.

5. N.N. Bogolyubov and Yu.A. Mitropolsky. Asymptotic Methods in the Theory of Nonlinear Oscillations. Gordon and Breach Science Publ., Vol. 537, New York, 1961. 
6. J.H.E. Cartwright, M. Feingold, and O. Piro. Global diffusion in a realistic three-dimensional time-dependent nonturbulent fluid flow. Phys. Rev. Lett., 75:3669-3672, 1995.

7. J.R. Cary, D.F. Escande, and J.L. Tennyson. Adiabatic-invariant change due to separatrix crossing. Phys. Rev. A, 34:4256-4275, 1986.

8. M. Feingold, L.P. Kadanoff, and O. Piro. Passive scalars, 3-dimensional volumepreserving maps, and chaos. J. of Statistical Physics, 50:529-565, 1988.

9. A.P. Itin, A.I. Neishtadt, and A.A. Vasiliev. Captures into resonance and scattering on resonance in dynamics of a charged relativistic particle in magnetic field and electrostatic wave. Physica D, 141:281-296, 2000.

10. I. Mezic and S. Wiggins. On the integrability and perturbation of threedimensional fluid flows with symmetry. J. Nonlinear Sci., 4:157-194, 1994.

11. Y. Nambu. Generalized hamiltonian dynamics. Phys. Rev. D, 7:2405-2412, 1975.

12. A.I. Neishtadt. Change of an adiabatic invariant at a separatrix. Sov. J. Plasma Phys., 12:568-573, 1986.

13. A.I. Neishtadt. On the change in the adiabatic invariant on crossing a separatrix in systems with two degrees of freedom. PMM USSR, 51:586-592, 1987.

14. A.I. Neishtadt. Scattering by resonances. Celestial Mechanics and Dynamical Astronomy, 111:1-20, 1997.

15. A.I. Neishtadt. On adiabatic invariance in two-frequency systems. In Hamiltonian systems with 3 or more degrees of freedom, NATO ASI Series C, volume 533, pages 193-213, 1999.

16. A.I. Neishtadt. Capture into resonance and scattering on resonances in twofrequency systems. In Proc. of the Steklov Institute of Mathematics, volume 250, pages 183-203, 2005.

17. A.I. Neishtadt, C. Simo, and A.A. Vasiliev. Geometric and statistical properties induced by separatrix crossings in volume-preserving systems. Nonlinearity, 16:521-557, 2003.

18. A.I. Neishtadt, D.L. Vainshtein, and A.A. Vasiliev. Chaotic advection in a cubic Stokes flow. Physica D, 111:227-242, 1998.

19. A.I. Neishtadt and A.A. Vasiliev. Change of the adiabatic invariant at a separatrix in a volume-preserving 3D system. Nonlinearity, 12:303-320, 1999.

20. T.H. Solomon and I. Mezic. Uniform, resonant chaotic mixing in fluid flows. Nature, 425:376-380, 2003.

21. H.A. Stone, A. Nadim, and S.H. Strogatz. Chaotic streamlines inside drops immersed in steady Stokes flows. J. of Fluid Mechanics, 232:629-646, 1991.

22. A.V. Timofeev. On the constancy of an adiabatic invariant when the nature of the motion changes. Sov. Phys. JETP, 48:656-659, 1978.

23. D.L. Vainchtein, A.I. Neishtadt, and I. Mezic. On passage through resonances in volume-preserving systems. Chaos, 16:art. \#043123, 2006.

24. D.L. Vainchtein, E.V. Rovinsky, L.M. Zelenyi, and A.I. Neishtadt. Resonances and particle stochastization in nonhomogeneous electromagnetic fields. J. of Nonlinear Science, 14:173-205, 2004.

25. D.L. Vainshtein, A.A. Vasiliev, and A.I. Neishtadt. Adiabatic chaos in a twodimensional mapping. Chaos, 6:514-518, 1996.

26. D.L. Vainshtein, A.A. Vasiliev, and A.I. Neishtadt. Changes in the adiabatic invariant and streamline chaos in confined incompressible Stokes flow. Chaos, 6:67-77, 1996. 
27. S.I. Vainshtein and Ya.B. Zel'dovich. Origin of magnetic fields in astrophysics. Sov. Phys. Usp., 15:159, 1972.

28. T. Ward and G.M. Homsy. Electrohydrodynamically driven chaotic mixing in a translating drop. II. Experiments. Physics of Fluids, 15:2987-2994, 2003.

29. J.B. Zeldovich, A.A. Ruzmaikin, and D.D. Sokolov. The representation of a 3-dimensional vector field by scalar potentials. Doklady akademii nauk SSSR, 284:103-106, 1985. 